https://doi.org/10.48009/1_iis_2005_296-302

\title{
AUTOMATED RUBRIC GENERATION AND ANALYSIS FOR ASSURANCE OF LEARNING TASKS
}

\author{
Dr. Michel Mitri, James Madison University, mitrimx@jmu.edu
}

\begin{abstract}
The AACSB now requires that assurance of learning (AOL) tasks in business schools must include course embedded assessments that directly measure student performance within the context of classes in the business core and/or major disciplines. The generally accepted format for presenting and documenting these assessments are rubrics. These rubrics include lists of learning objectives and ratings to assign for selected students on certain projects and/or examinations. Rubric data is then collated and reported for the purpose of identifying performance trends, ultimately leading to course and program improvements. This can be a very labor-intensive process, and information technology may be useful to reduce manual and time consuming operations. This paper presents an Excel macro that assists in automating the process of generating rubrics, scoring student performance, and summarizing assessment results.
\end{abstract}

Keywords: AACSB, ABET, assurance of learning, course embedded assessment, learning objectives, Excel macros, rubrics

\section{INTRODUCTION}

The Association to Advance Collegiate Schools of Business (AACSB) has established specific requirements for measuring educational effectiveness in business programs. Specifically, standards 16, 18, 19, and 21 mandate creation and measurement of learning goals in undergraduate, general masters, specialized masters, and doctoral programs, respectively [1]. These assurance of learning (AOL) standards serve two main purposes. First, they "demonstrate that accountability (such as in accreditation) is an important reason to assess learning accomplishments. Measures of learning can assure external constituents such as potential students, trustees, public officials, supporters, and accreditors, that the organization meets its goals" [1]. Second, they "assist the school and faculty members to improve programs and courses." [1]. Other accrediting agencies have adopted similar requirements. Of particular relevance to IS programs, the Accreditation Board for Engineering and Technology (ABET) Computing Accreditation Commission (CAC) includes IS standards I-1 through I-5, which address the need for establishing learning objectives, measuring results, and using assessment results to guide in curriculum improvement.

While recognizing the differences in mission of various institutions, AACSB and ABET maintain that all schools must identify learning goals, and must measure student outcomes with respect to these goals. In the past, indirect measures such as surveys and focus groups were sufficient for this purpose, but the current standards mandate that direct measures of student performance must also be used in order to meet the assurance of learning (AOL) requirements. 
AACSB identifies two direct assessment methods, stand-alone testing and course-embedded measures. Collection and analysis of stand-alone testing is fairly straightforward, as it generally involves multiple-choice tests that can easily be collated and organized using computerized tools. Course-embedded assessment is a bit more problematic, and often involves a labor-intensive process instructor involvement. Often this involves evaluating written and oral presentations, and therefore introduces evaluator subjectivity. Furthermore, data collection and summarization is more difficult when dealing with tacit, qualitative evaluations $[4,5]$.

This paper presents a tool that helps to simplify the tasks involved in course-embedded assessment. The tool is an Excel workbook containing macros that automate the process of generating rubrics, evaluating student performance, and summarizing results. The outputs from this tool can be easily embedded into assessment reports, and are useful for identifying necessary program improvements.

\section{MANUAL TECHNIQUES FOR COURSE EMBEDDED ASSESSMENT}

The primary tool recommended for implementing course-embedded assessment is called a rubric. A rubric is a "description of the standards that will be used to judge a student's work on each of the criteria or important dimensions of learning. It is a scoring guide that is used in subjective appraisals of student work. It makes explicit statements about the expected qualities of performance at each point on a scale" [6].

Rubrics are typically implemented as paper-based documents, and are filled manually by the evaluators. Rubrics vary in content and format, but they all include the following characteristics: (1) a list of learning objectives, (2) a set of ratings (and associated descriptions) that identify the evaluation scale for each learning objective, (3) a table format that includes cells for each possible rating of each objective (which the instructor will check based on his or her evaluation of the student with respect to the particular objective), and (4) a location for the instructor to include comments.

Once rubrics are filled, the data must be collected and summarized. If rubrics have been filled manually using pencil-and-paper, this can be an onerous and error-prone task. Either data collection and summarization is done manually, where average scores and rating totals are calculated by hand, or an additional data entry process must be done in order to transfer the data from rubrics into spreadsheets or databases, accompanied by construction of the necessary formulas, queries, and charts for reporting purposes.

Thus, information technology is a promising venue for easing the burden of rubric creation, student evaluation, data summarization, and assessment reporting. The following sections describe an automated rubric generator for assisting with these tasks. 


\section{DESCRIPTION OF THE AUTOMATED RUBRIC GENERATOR}

The automated rubric generator is implemented as an Excel workbook, which will be referred to as Rubric Creator in this paper. Rubric Creator contains a Control Sheet with an associated macro [2]. Within a control sheet, the user provides the following information: the instructor (evaluator), the students being evaluated, a list of topics (content areas) for which students are being evaluated, a list of skills that are to be applied to these topics, and a list of ratings and associated scores that will be used to gauge student performance. A sample Control Sheet is shown in Figure 1.

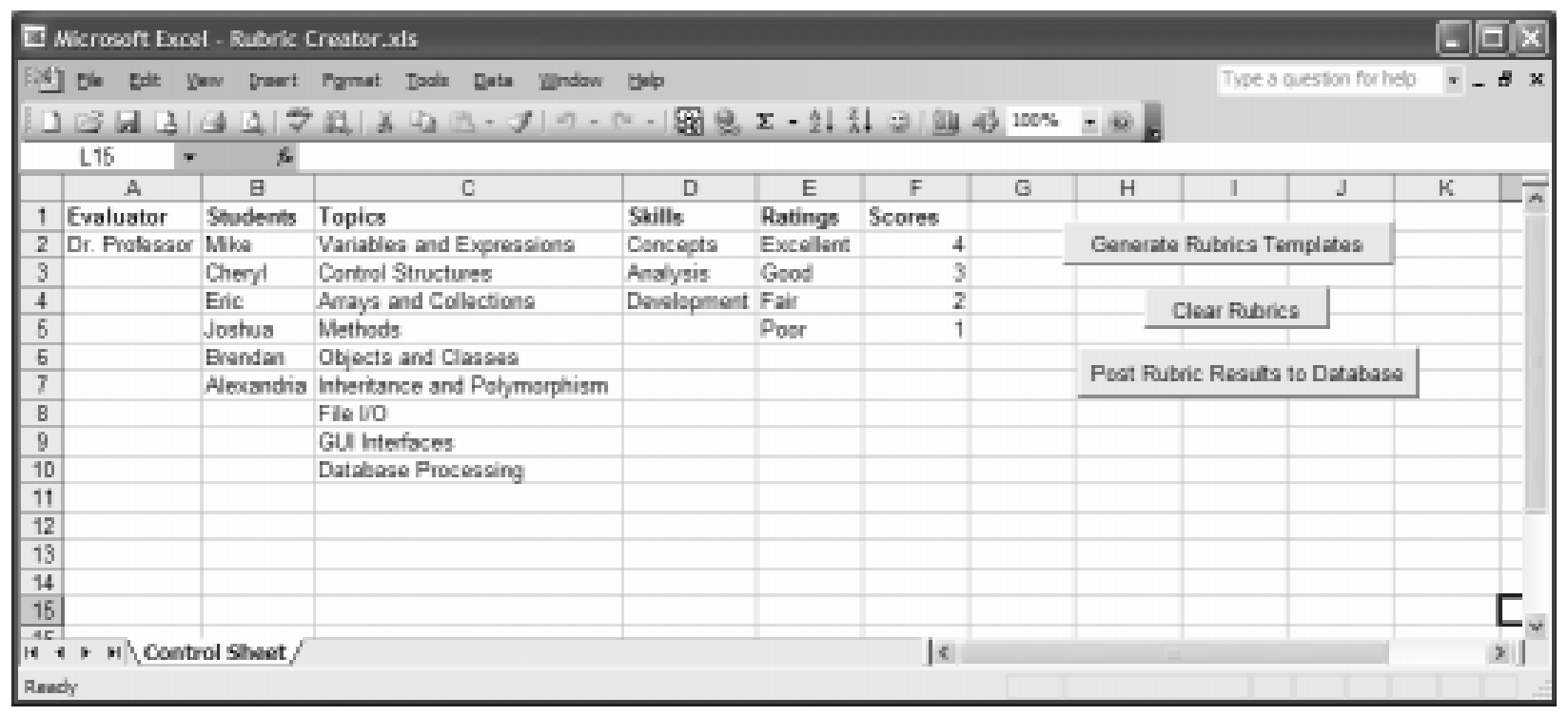

Figure 1. Control Sheet of Rubric Creator

In this example, rubrics will be created for an intermediate Java programming course. Students are to be evaluate on their performance in three main skills: (1) concepts (terminology and definitions of programming constructs), (2) analysis (the ability to look at existing program code and determine the program behavior and memory contents that will result from executing the program), and (3) development (the ability to write programs to meet functional specifications). The evaluations will be given for application of these skills to the nine major topics covered in the course, as shown in column $\mathrm{C}$ of the control sheet. For each evaluation, the instructor can give a rating of excellent, good, fair, or poor, and the associated scores that will be used for calculating student performance will be $4,3,2$, and 1 , respectively.

Note that the control sheet allows the user to specify ANY evaluator, students, topics, skills, ratings and scores. Rubrics will be generated based on the values entered by the user in the control sheet. 


\section{INVOKING THE RUBRIC GENERATION MACRO}

Note that there are three buttons on the Control Sheet. When the user clicks the Generate Rubric Templates button, rubric templates will be generated for each student. Each rubric template is implemented as a new sheet in the workbook; these sheets are automatically created by the Excel macro. A sample rubric template sheet is shown in Figure 2.

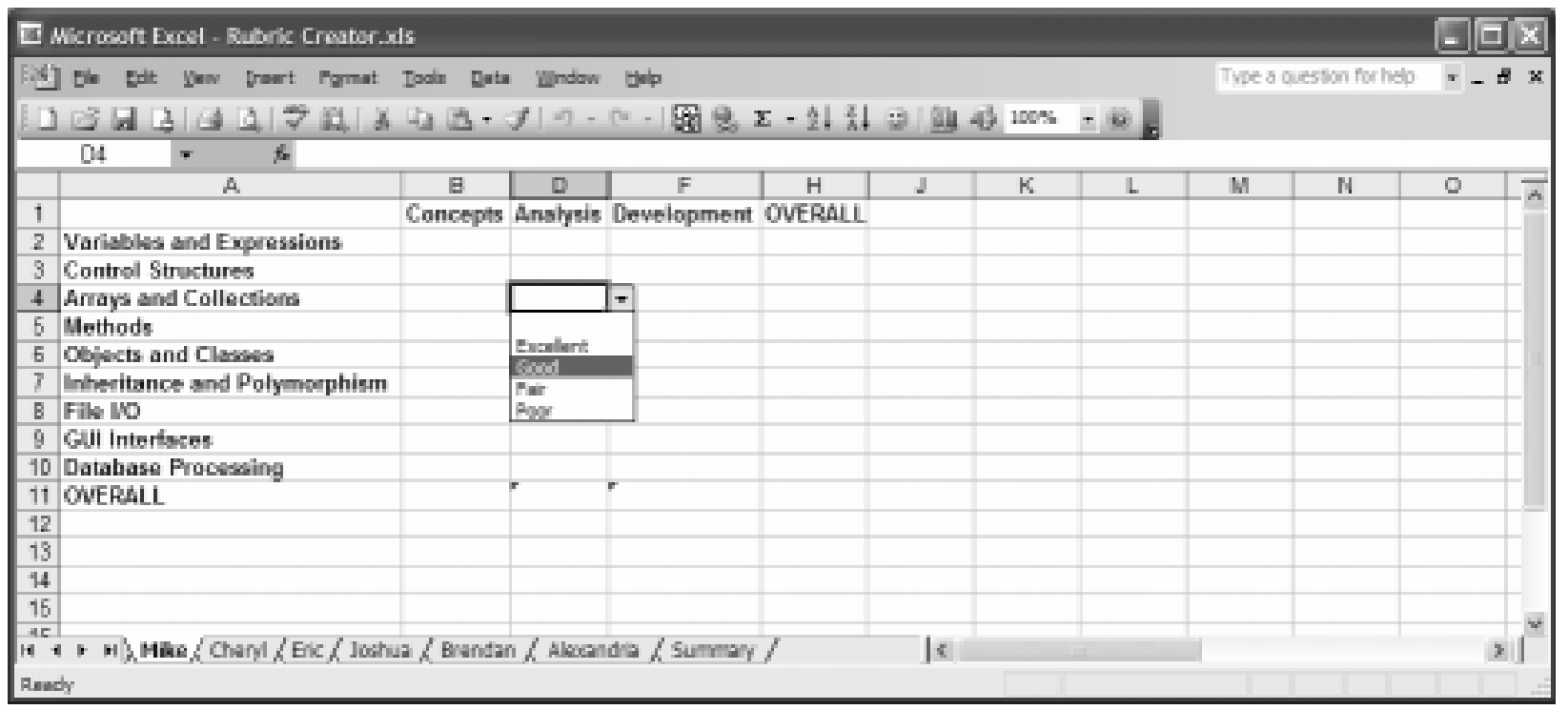

Figure 2. Rubric Template Sheet for a Student

Each rubric sheet displays the topics in rows and the skills in columns. Each cell is equipped with a dropdown list that allows the user to select a rating for the application of a skill to a topic. For example, in figure 2, the cell D4 will contain the evaluator's rating for the student's performance in analyzing code with respect to arrays and collections. As you can see, in this example with nine topics and three skills, there are a total of twenty seven ratings that can be applied to a student in this rubric. In general, the number of evaluations that a rubric contains is equal to the number of topics times the number of skills that the evaluator specified in the control sheet.

As described earlier, the typical manual approach in rubric use is to check the appropriate rating box for each objective in a paper-based rubric form. With the Excel rubric template sheet, the user selects from the dropdown list for each evaluation cell in the sheet. On a paper-based rubric, there is typically space in which to jot down comments for each objective. Similarly, in the Excel rubric sheet, the user can insert a comment into any cell of the rubric template. These are shown in Figure 3.

One advantage of the Excel rubric template is that it automatically calculates overall ratings for each topic (across skills), for each skill (across topics), and the overall score for the student (see Figure 3). This is made possible because the macro generates the calculation procedures for summarizing scores and ratings for each rubric template. 


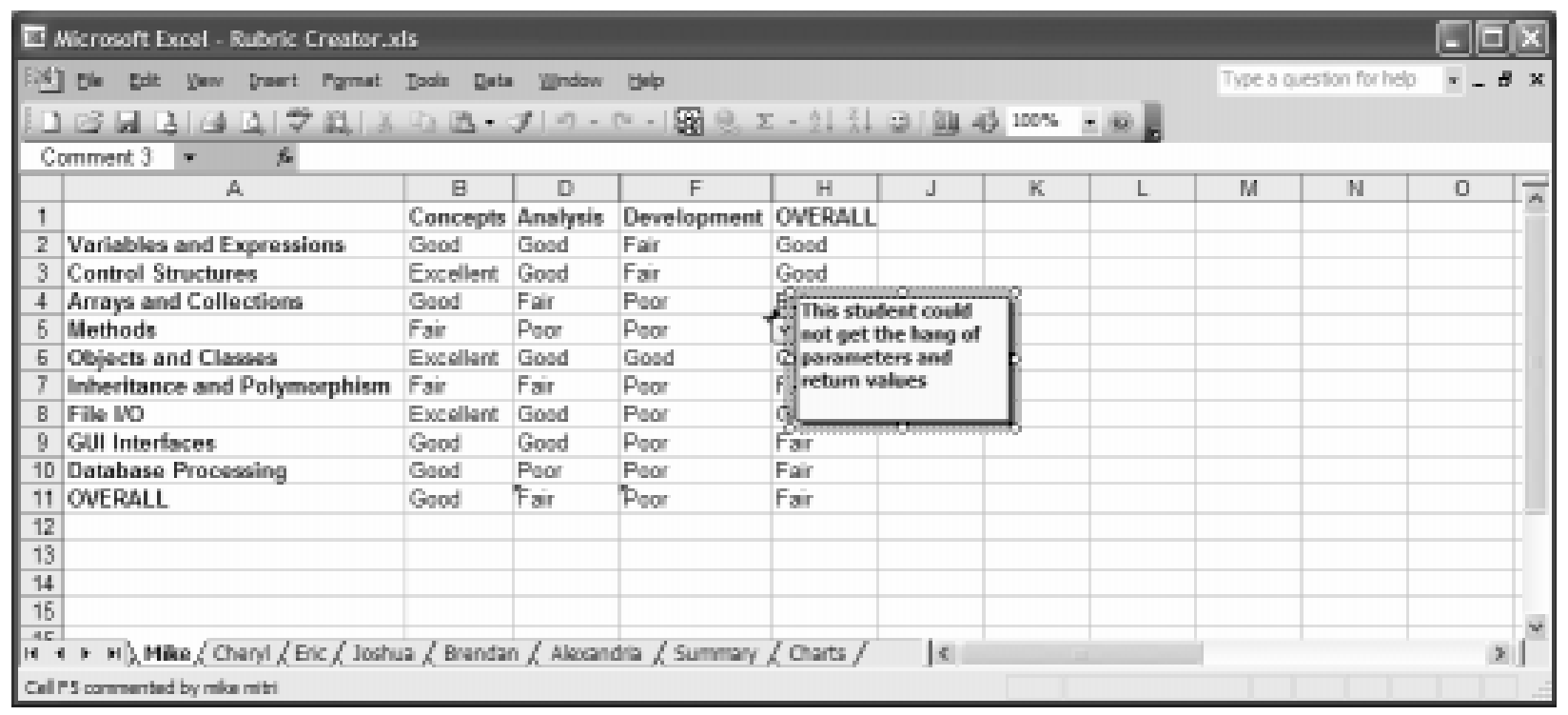

Figure 3. Rubric Template Filled out, With Comments and Calculated Overall Ratings

\section{SUMMARIZING THE RESULTS}

The final sheet created by RubricCreator's rubric generation macro is a summary sheet that includes formulas for calculating assessment averages and totals. This sheet is shown in Figure 4.

The summary sheet automatically calculates and displays the overall results of the rubric assessment. Specifically, average ratings are calculated and displayed for each topic/skill combination. In addition, the percent of students who obtained each overall rating for each skill is displayed in both numeric form and in a bar chart. Similarly, the percent of students who obtained each overall rating by topic is also displayed textually and graphically.

Users can easily copy and paste portions of the summary sheet into their assessment report documents. 


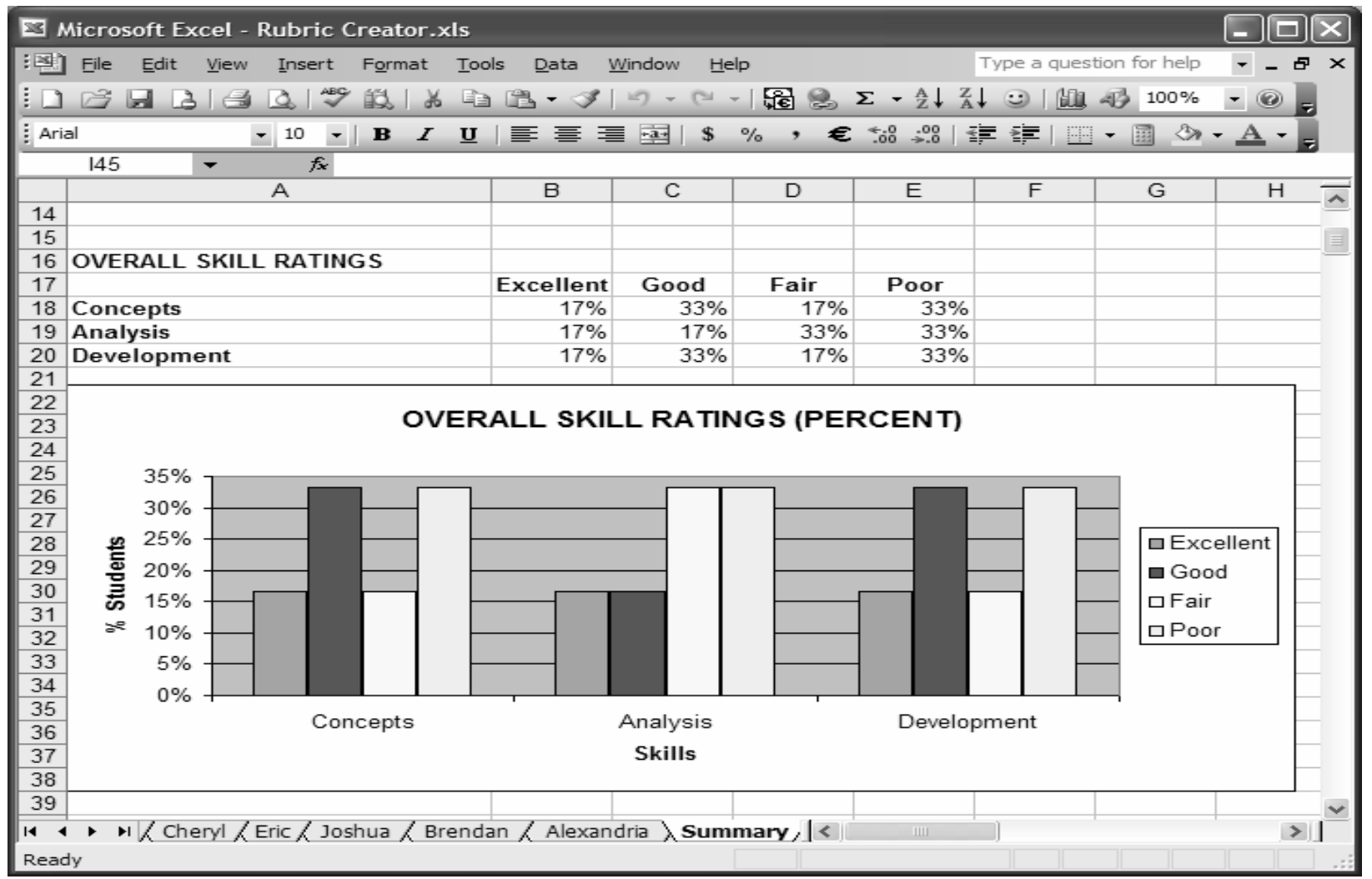

Figure 4. Summary Sheet Showing Overall Trends of Assessment Result

\section{POSTING TO THE DATABASE}

The Rubric Creator's Control Sheet includes a button for posting results to a database. The database in question is a Microsoft Access database that stores time-stamped judgments indexed by evaluator, student, topic and skill. Rubric evaluations and their comments are automatically downloaded to the database when the button is clicked.

This database includes comprehensive functionality enabling users to view assessment trends over time, and filtered by any combination of factors including students, majors, evaluators, programs, date ranges, topics, and/or skills. In addition, the database includes an artificial intelligence component that utilizes semantic network and multi-attribute utility techniques for enabling inferential predictions of student performance based on existing judgments. While a detailed description of the database is beyond the scope of this article, more information can be found in $[4,5]$.

\section{CONCLUSION}

There is increasing demand for course embedded direct assessment in business and technology university programs. Traditional assessment methods like surveys and standardized tests are no longer sufficient to satisfy accrediting agencies. This results in increased burden on faculty for 
qualitative evaluation of a variety of skills (including written and oral communication) in a variety of academic subjects (including all the usual business majors). Rubrics are the accepted methodology for implementing these evaluations, but the manual rubric process makes data collection and summarization difficult. Information technology should be brought to bear to facilitate this process.

This paper described one attempt at automating the rubric-oriented assessment approach. The technique involves an Excel workbook with embedded macros for automatically generating rubric templates, providing a simple user interface for filling the rubrics with ratings and comments, and accumulating/summarizing assessment results. The use of this technology can potentially save considerable time and effort for faculty in their assessment process.

\section{REFERENCES}

1. AACSB Assessment Resource Center: http://www.aacsb.edu/resource_centers/assessment/standards.asp.

2. ABET CAC Standards: http://www.abet.org/info_prgs_cac.html, http://www.abet.org/documents/cac/C4\%2003-04\%20IS\%20SelfStudy\%20Questionnaire\%208-7-02.doc.

3. Bluttman, K. (2004) Developing Microsoft Office Solutions. Boston, MA: Addison Wesley, Pearson Education.

4. Mitri, M. (Fall 2003). Applying Knowledge Management Techniques for Performance Assessment. Computers and Education, 41.

5. Mitri, M. (Summer 2003). A Knowledge Management Framework for Curriculum Assessment. Journal of Computer Information Systems, 43(4).

6. New Horizons for Learning. http://www.newhorizons.org/strategies/assess/terminology.htm 